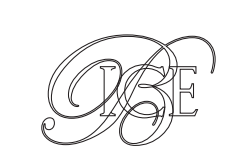

\title{
EL POTENCIAL EFECTO COMPETITIVO DE LAS ESTACIONES DE SERVICIO AUTOMÁTICAS EN ESPAÑA
}

El objeto de este estudio es el análisis de las estaciones de servicio automáticas, que potencialmente tienen capacidad de aumentar la presión competitiva. No obstante, en los últimos años se observan restricciones normativas a nivel nacional y autonómico ante la libre operación de este tipo de instalaciones. Mediante la utilización de una metodología econométrica de diferencias en diferencias, se cuantifican los efectos para el caso concreto de la Comunidad Autónoma de Madrid, tomando como referencia el estudio de la CNMC sobre estaciones de servicio automáticas realizado en julio de 2019. Se confirma que las estaciones automáticas aumentan la competencia en una isócrona de 10 minutos en su entorno, y especialmente lo hacen las instalaciones nuevas reduciendo los precios de gasóleo A y de gasolina 95 de las estaciones atendidas y operadas por empresas independientes en un 3,5\% y un 2,9\%, respectivamente. Además, se observa que las verticalmente integradas tienen una reacción mucho más reducida que las independientes.

Palabras clave: carburantes, política de competencia, regulación.

Clasificación JEL: L4, L71, D43, K21.

\section{Introducción}

En la última década, España ha observado un aumento paulatino de la apertura de estaciones de servicio (EE SS) automáticas. Estas instalaciones, también llamadas desatendidas o sin personal (unmanned gas stations, según la literatura anglosajona), se caracterizan por

\footnotetext{
* Técnico de la Comisión Nacional de los Mercados y la Competencia en la Subdirección de Análisis Económico (Departamento de Promoción de la Competencia).

La autora agradece los comentarios y aportaciones de María Vidales Picazo, Héctor Jiménez Portilla y Pablo Delgado Cubillo.

Versión de abril de 2020

DOI: https:/doi.org/10.32796/bice.2020.3126.7071
}

no disponer de personal propio para realizar el repostaje y el cobro a los clientes, sino que es el cliente quien se suministra carburante y realiza el pago mediante banca online, a diferencia de las instalaciones tradicionales (EE SS atendidas, donde el repostaje lo realiza personal de la propia instalación, y estaciones de autoservicio, donde el repostaje es realizado por el cliente pero hay al menos un operario en la instalación encargado de activar el surtidor y realizar el cobro).

Otros países europeos, en especial los nórdicos, han experimentado esta tendencia creciente en décadas anteriores y, actualmente, $D$ 
han alcanzado una penetración notable en el mercado de estaciones de servicios. En Dinamarca las instalaciones automáticas representaban, en 2018, el $75 \%$ del total de EE SS (Drivkraft Danmark, 2019) y en Suecia, el $67 \%$ ese mismo año (SPBI, 2019).

La aparición de EE SS automáticas, por un lado, implica la entrada de agentes con una estructura de costes diferente (debido a menores costes operativos) con respecto a las estaciones tradicionales, y, por otro lado, aumenta el número y la variedad de los puntos de venta de carburantes de automoción. Además, las EE SS automáticas ofrecen mayor flexibilidad en el mercado minorista de carburantes, especialmente en zonas rurales de poco tráfico y también en zonas urbanas vinculadas a negocios ya existentes o de nueva creación. De esta forma, estas EE SS reúnen características de mavericks ${ }^{1}$, creando potencialmente mayor presión competitiva sobre los precios en los mercados locales.

La mayor presencia de las EE SS automáticas se explica por los avances tecnológicos y por los incentivos indirectos incluidos en el RDL 4/2013, que fomentaba la instalación de estaciones independientes, y, entre ellas (indirectamente) automáticas, en establecimientos comerciales y en polígonos industriales, entre otros. No obstante, existe regulación regional y nacional en España que impone restricciones a la apertura y operación de nuevas EE SS automáticas, como ha señalado la CNMC (2016, 2019) en varias ocasiones.

El presente estudio busca analizar los efectos competitivos de la entrada de las EE SS automáticas en el mercado de distribución de

1 Un maverick o "empresa díscola» es una empresa que ejerce una importante presión competitiva en los mercados al tener un papel disruptivo respecto a las conductas de los operadores incumbentes, lo que muy probablemente permitirá romper una potencial conducta coordinada (US Department of Justice \& Federal Trade Commission, 2010). carburantes, y en particular pretende cuantificar dichos efectos para el caso concreto de la Comunidad Autónoma de Madrid (CAM), tomando como referencia el estudio de la CNMC sobre EE SS automáticas realizado en julio de 2019 (en adelante el Estudio).

El presente artículo se estructura del siguiente modo: en primer lugar, se describe el mercado minorista de distribución de carburantes en España; en segundo lugar, se analiza el marco regulatorio a nivel nacional y autonómico; en tercer lugar, se presenta la cuantificación econométrica de los efectos de la aparición de EE SS automáticas en la CAM con base en el Estudio y, por último, se presentan las conclusiones.

\section{Descripción del mercado}

A principios de 2020, en el mercado de distribución de carburante en España, existen más de 11.600 EE SS, una cifra que es el resultado de un crecimiento constante en los últimos años, como se observa en el Gráfico 1. El aumento total en el número de EE SS es de un $14 \%$ entre 2013 y 2020.

Descomponiendo por tipo de operador, el Gráfico 2 muestra diferencias sustanciales en la evolución del número de EE SS. En el año 2016 se produce el mayor crecimiento del periodo estudiado, ya que se produjo un aumento notable de las EE SS independientes (en torno a un $9 \%$ ) y un crecimiento muy reducido, cercano al $0,5 \%$, en el caso de las EE SS verticalmente integradas y las otras abanderadas. En otros años esta relación es parecida, con la diferencia de que las EE SS independientes crecen algo menos, pero por encima de un $4 \%$ anual, mientras las verticalmente integradas experimentan ligeros decrecimientos (cercanos al $1 \%$ ). El resto de estaciones $\square$ 

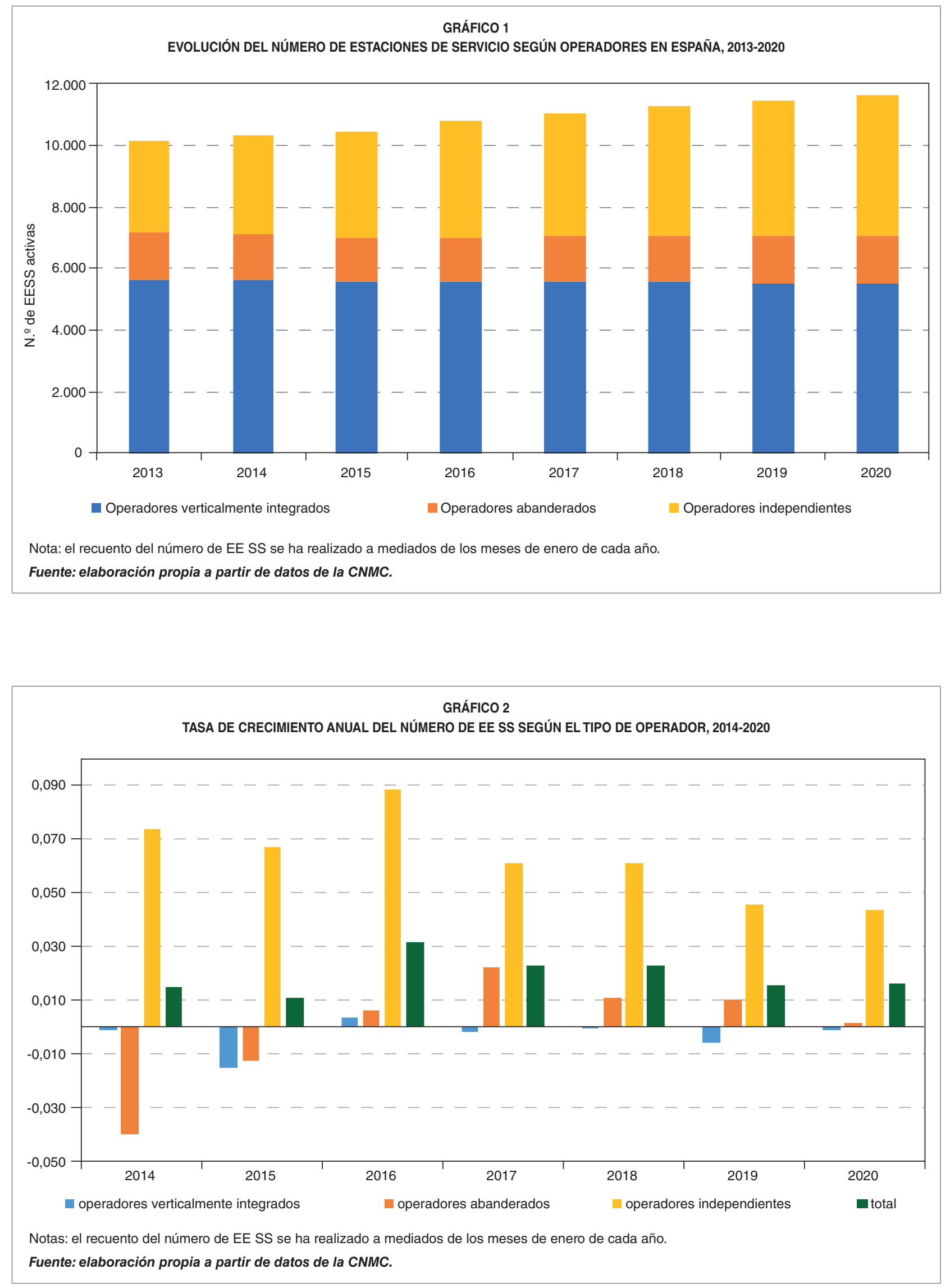
Gabriella Németh Kecskeméti

GRÁFICO 3
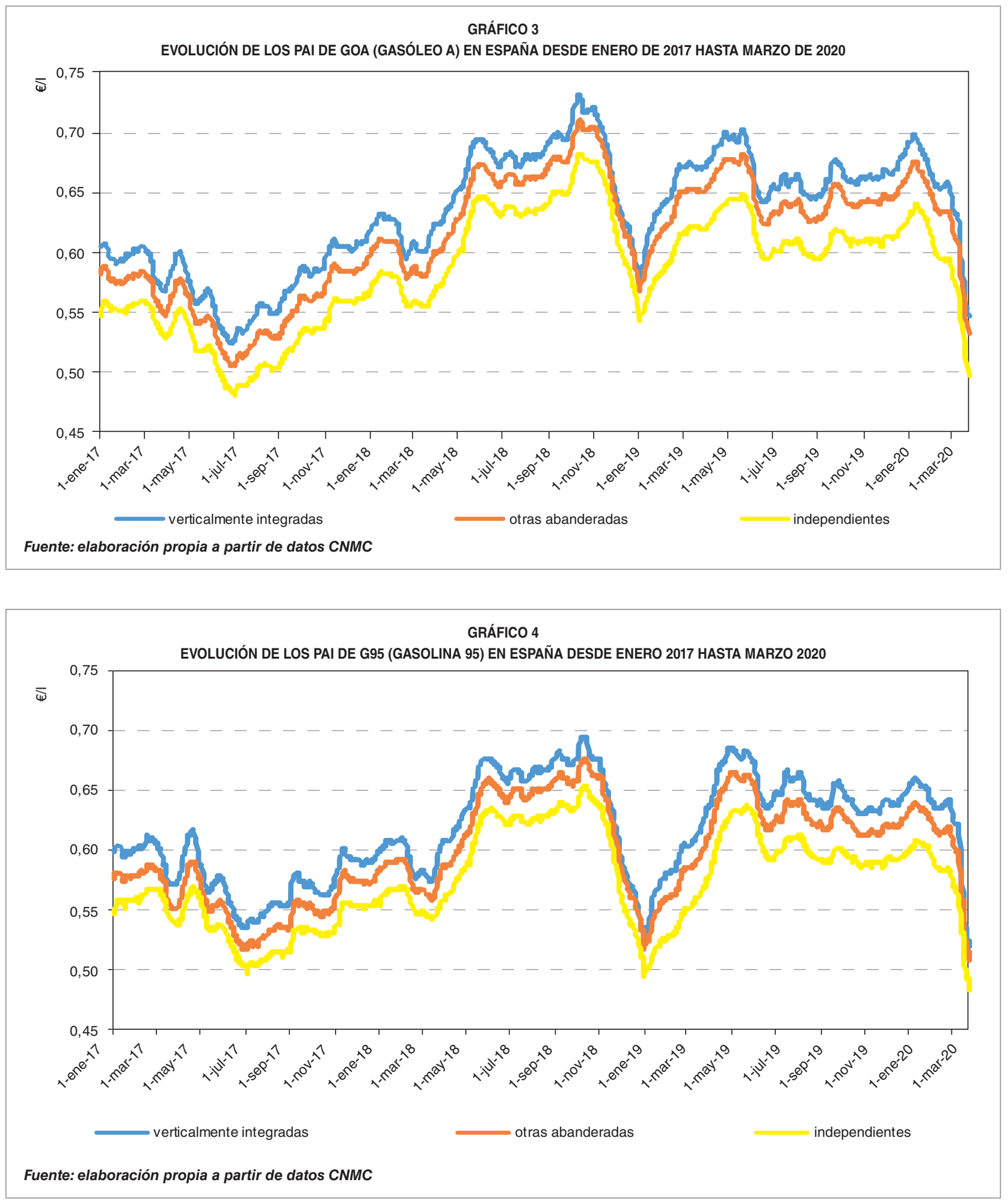

abanderadas crecen a tasas del $1-2 \%$ anual en el conjunto del periodo.

Teniendo en cuenta la evolución de los precios antes de impuestos (PAI) de los dos principales tipos de carburantes según los diferentes tipos de EE SS en los últimos años (2017-2020), se observa que las EE SS verticalmente integradas tienen precios antes de $D$ 
impuestos más altos que las EE SS abanderadas y las independientes en el conjunto de España, como así lo muestra el Gráfico 3.

\subsection{Las estaciones automáticas}

La principal característica distintiva de las EE SS automáticas es que el repostaje físico lo realiza el cliente directamente, previo pago electrónico ${ }^{2}$. En el presente estudio, la distinción entre las EE SS automáticas nuevas y reconvertidas $^{3}$ es fundamental, ya que permite discernir sus potenciales efectos como mavericks. Las automáticas nuevas pertenecen mayoritariamente a grupos independientes, no tienen contratos de

2 Reglamento de instalaciones petrolíferas, aprobado por el Real Decreto 2085/1994, de 20 de octubre, e instrucciones técnicas complementarias MI-IP 03, aprobada por el Real Decreto 1427/1997, de 15 de septiembre, y MI-IP 04, aprobada por el Real Decreto 2201/1995, de 28 de diciembre. Solo se consideran estaciones de servicio desatendidas aquellas que no tengan ningún personal afecto a su instalación durante todo el horario de apertura (24h).

3 Se consideran EE SS automáticas nuevas aquellas que han estado operando como automáticas desde sus comienzos, mientras que las EE SS automáticas originalmente operaban como atendidas y, en un momento dado, sus operadores decidieron reconvertirlas en automáticas. suministro en exclusiva, ni de gestión, y, por tanto, tienen menos condicionantes a la hora de fijar precios que las automáticas reconvertidas. Sin embargo, las automáticas reconvertidas, cuyos operadores son mayoritariamente empresas verticalmente integradas o abanderadas, pueden adoptar una estrategia empresarial que tenga por objetivo acaparar posiciones en el mercado y adelantar y/o expulsar a estaciones automáticas rivales de los mercados locales.

Actualmente, el registro de las EE SS automáticas lo llevan a cabo las CC AA, por lo que a nivel nacional no existen datos públicos precisos de fuentes oficiales respecto al número y ubicación de las EE SS automáticas en España. Según datos de la Asociación Nacional de Estaciones de Servicio Automáticas (AESAE), en marzo de 2019 existían 882 EE SS automáticas independientes en España ${ }^{4}$, a las que habría que añadir las EE SS automáticas $D$

4 Los principales operadores de estas EE SS son Ballenoil, Petroprix, Gasexpress, Settram, Plenoil, BonArea, Autonet\&Oil, Naftë, Petrocar, Low Gas, Nubex, Sorval, Eureka y Moove Low Cost.

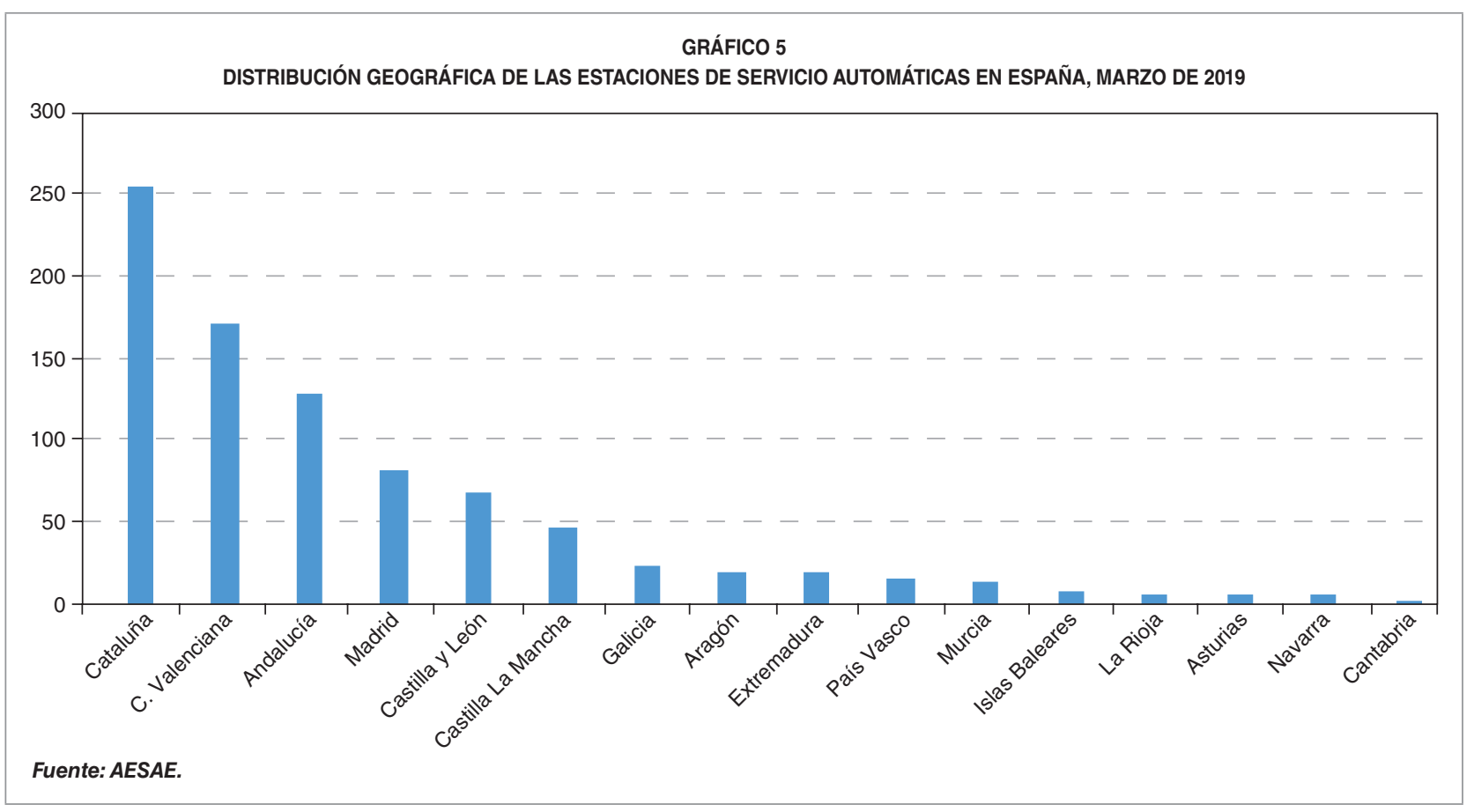


operadas por empresas verticalmente integradas y de otras abanderadas.

Los datos de AESAE muestran que las estaciones de servicio automáticas están muy concentradas en unas pocas CC AA. Así, en Cataluña, Comunidad Valenciana, Andalucía y Madrid se ubicaban en 2019 el $72 \%$ de las EE SS automáticas en España. En el otro extremo, cabe resaltar la baja penetración de EE SS automáticas en las CC AA del norte peninsular (Galicia, Cantabria, Asturias, Navarra, La Rioja, Aragón), Extremadura y Baleares.

\section{Regulación de las estaciones de servicio automáticas}

La normativa estatal de aplicación a las estaciones de servicio (sean o no automáticas) es la Ley 34/1998, de 7 de octubre, del sector de hidrocarburos, la cual fue modificada por el artículo 39 de la Ley 11/2013, de 26 de julio, de medidas de apoyo al emprendedor y de estímulo del crecimiento y de la creación de empleo. De esta forma, el artículo 43.2 de la Ley 34/1998 establece que «la actividad de distribución al por menor de carburante y combustibles petrolíferos podrá ser ejercida libremente por cualquier persona física o jurídica» y que «las instalaciones utilizadas para el ejercicio de esta actividad deberán cumplir con los actos de control preceptivos para cada tipo de instalación, de acuerdo con las instrucciones técnicas complementarias que establezcan las condiciones técnicas y de seguridad de dichas instalaciones, así como cumplir con el resto de la normativa vigente que en cada caso sea de aplicación, en especial la referente a metrología y metrotecnia y a protección de los consumidores y usuarios».

Por tanto, de cara a conocer los requisitos concretos que han de cumplir las EE SS automáticas, es necesario acudir a la normativa específica a nivel técnico y de seguridad.

En lo que respecta a la normativa relativa a metrología, los requisitos esenciales establecidos en el Real Decreto 244/2016, de 3 de junio, por el que se desarrolla la Ley 32/2014, de 22 de diciembre, de Metrología hacen referencia a las características técnicas de los surtidores y son los mismos para las tres categorías de estaciones de servicio existentes, pudiéndose cumplir independientemente de la existencia o no de personal en la estación de servicio (CNMC, 2016).

Sin embargo, para garantizar la seguridad y la protección de consumidores y usuarios, la regulación a nivel nacional de las estaciones de servicio automáticas es más estricta y tiene requisitos adicionales respecto a las otras dos modalidades de instalaciones (atendidas y en autoservicio). En concreto, el Real Decreto 706/2017, de 7 de julio, por el que se aprueba la instrucción técnica complementaria MI-IP 04 «Instalaciones para suministro a vehículos» y se regulan determinados aspectos de la reglamentación de instalaciones petrolíferas ${ }^{5}$, establece en su capítulo 13 las siguientes medidas de seguridad y comunicación que deben cumplir las estaciones de servicio automáticas.

Medidas adicionales de seguridad:

- Obligación de eliminar el mecanismo que fija el boquerel en la manguera de suministro.

- Obligación de limitar el tiempo de cada suministro a tres minutos y a un volumen total de 75 litros.

5 Adicionalmente, en enero de 2019, el Ministerio de Industria, Comercio y Turismo publicó la Guía Técnica de Aplicación Práctica de la Instrucción Técnica Complementaria MI-IP de Instalaciones para el Suministro de Vehículos que se puede consultar en el siguiente enlace www.f2i2.net/documentos/Isi/InstPetroliferas/Guia_Aplicacion_IP_04_2019. pdf. 
- Disponer de un certificado del sistema de protección contra incendios adecuado a los nuevos requisitos de operación de la instalación.

- Protección de todas las arquetas de la instalación contra un acceso no autorizado para evitar su apertura y manipulación.

- Disponer de un sistema de comunicación bidireccional a un centro de control propio o ajeno, donde se pueda solicitar ayuda, transmitir instrucciones y atender las incidencias y emergencias.

- Disponer de un circuito cerrado de televisión con grabación y transmisión de imágenes que permita ver la operación desde un centro de control remoto.

- Disponer de un sistema de monitorización con acceso remoto desde el centro de control para la recepción de alarmas y la supervisión de los principales equipos de la instalación.

- Disponer de un interruptor de paro de emergencia claramente visible.

- Disponer de equipos automáticos de detección y extinción de incendios en cada punto de suministro.

Obligaciones adicionales de comunicación e inspección:

- Comunicar el carácter desatendido de la instalación al órgano competente en materia de industria de la comunidad autónoma previamente a la puesta en marcha.

- Informar al cliente del carácter desatendido de la instalación a través de un cartel en la entrada que sea visible desde el interior del vehículo.

- Disponer de un número de teléfono de emergencias con atención 24 horas.
- Disponer de un protocolo de actuación para el caso de que las descargas de camiones cisterna se produzcan también sin asistencia de personal de la instalación.

- Disponer de un procedimiento de inspección periódica de los equipos de trabajo y seguridad.

Por último, la apertura de una estación de servicio automática tiene también que cumplir con la normativa específica desarrollada por la comunidad autónoma donde preste el servicio. Es precisamente en este punto donde tradicionalmente se han venido incluyendo las principales limitaciones a la apertura de este tipo de instalaciones. Según la CNMC (2016), la principal restricción radica en la presencia obligatoria de al menos una persona responsable de los servicios que se prestan en la estación de servicio y/o responsable de la supervisión de la maniobra de repostaje. Es decir, hay CC AA, como, por ejemplo, Andalucía, CastiIla-La Mancha, Canarias, Murcia ${ }^{6}$ y La Rioja donde se imposibilita de facto la existencia de estaciones de servicio automáticas.

Por otro lado, existen otras medidas obligatorias en varias CC AA que, si bien no requieren de manera directa la presencia de una persona, sí lo hacen de forma indirecta para poder cumplir con otras obligaciones de mantenimiento de la instalación. Se trata, por ejemplo, de disponer de hojas de reclamaciones en todo momento, en lugar de ofrecer la posibilidad de poner la reclamación por medios telemáticos; la necesidad de tener a disposición de los $\triangle$

6 En el caso de Murcia, el 21 de febrero de 2020, se aprobó un decreto ley que eliminaba la obligación de tener personal afecto a las instalaciones, si bien, dicho decreto ley quedó tácitamente derogado al no haberse celebrado el debate sobre su convalidación por haberse decretado el estado de alarma, por lo que la restricción sigue estando en vigor. 
usuarios el recipiente de medición, certificado y calibrado oficialmente, en lugar de solamente obligar a tenerlo a disposición de los servicios de Inspección de la Administración; o la obligación de colocar carteles de fuera de servicio en caso de que los surtidores, los medidores de presión o los dispositivos suministradores de agua dejen de funcionar.

En conclusión, la normativa que regula las estaciones de servicio automáticas debe establecer las medidas necesarias para que el servicio de repostaje se preste con garantías de seguridad y de protección de los usuarios y consumidores. Ahora bien, todas aquellas restricciones adicionales que suponen de manera directa o indirecta la obligación de prestar un servicio atendido deberían ser eliminadas porque no se ajustan a los criterios de necesidad y proporcionalidad, y no benefician a los consumidores ni en términos de precios ni de seguridad ni de calidad del servicio (CNMC 2016 y 2019).

\section{Cuantificación del efecto competitivo de las EE SS automáticas en mercados locales: ejemplo de la Comunidad de Madrid}

En el mercado minorista de carburantes, las EE SS atendidas ofrecen servicios adicionales y, en consecuencia, sus costes son más elevados que los de las automáticas. Por este motivo, los precios de las atendidas también tienden a ser superiores. No obstante, estos dos tipos de EE SS actúan en los mismos mercados y compiten en precios, como constata la Comisión Europea (2008, 2016a y 2016b).

En esta misma línea se expresa el estudio Análisis del efecto competitivo de la entrada de gasolineras automáticas en el mercado de distribución minorista de carburantes, publicado por la CNMC en julio 2019, que centra su análisis en la Comunidad Autónoma de $D$

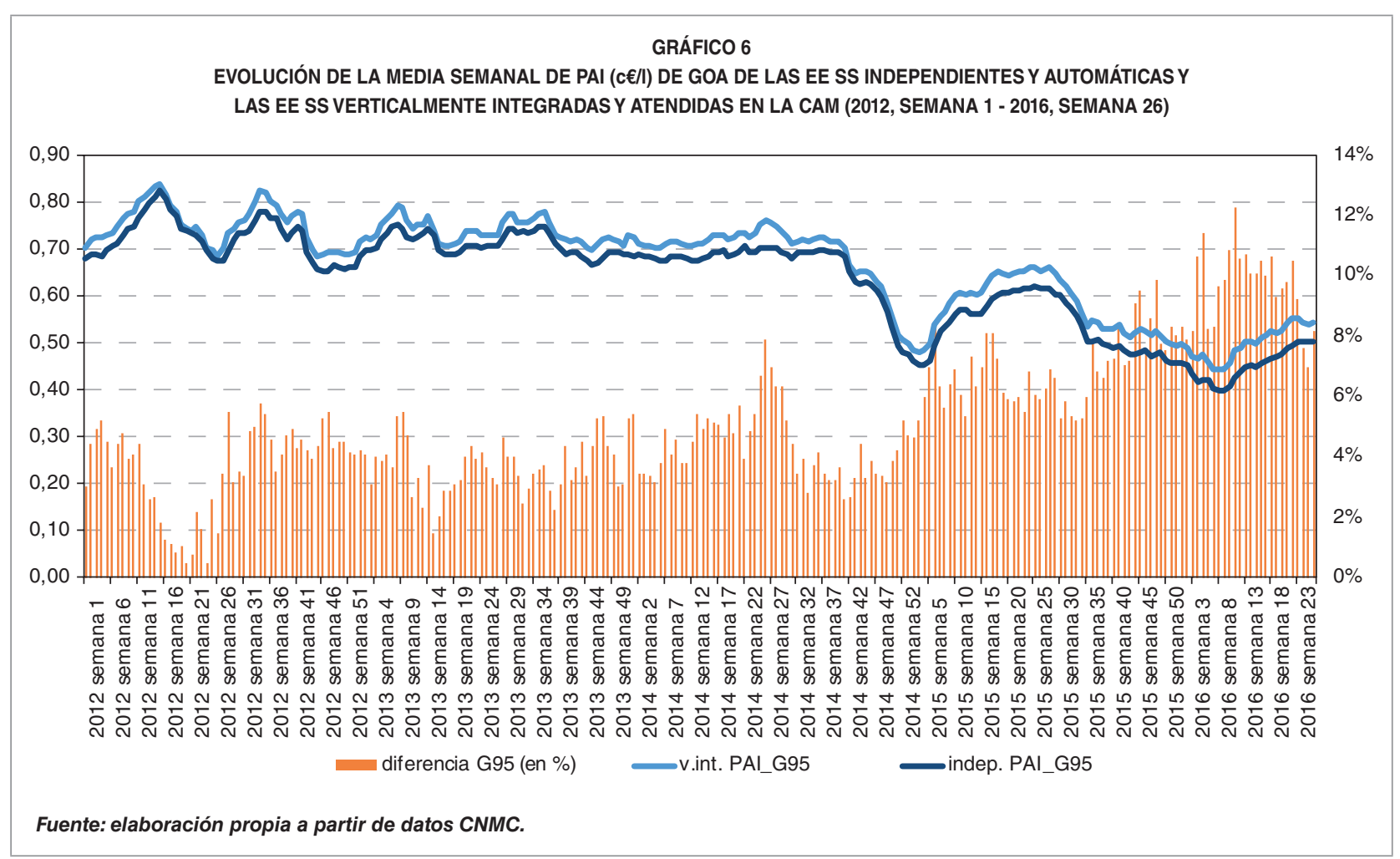


Madrid (CAM), señalando la evolución de los precios entre, por un lado, aquellas estaciones automáticas que están operadas por independientes, y, por otro lado, aquellas estaciones atendidas operadas por empresas verticalmente integradas. En el periodo estudiado entre mediados de 2011 y mediados de 2016, la diferencia de los precios entre estos dos conjuntos de instalaciones va aumentando conforme van entrando más estaciones automáticas en esta comunidad autónoma; esto es, a partir del segundo semestre de 2015, cuando las estaciones automáticas alcanzaron una penetración sobre el total de un $6 \%$, duplicando su nivel del año anterior, anterior, y en 2016 llegaron a un nivel de aproximadamente un $9 \%$. La diferencia de precios de GOA se situó en torno a un $17 \%$ y de G95, cerca de un $12 \%$ a principios de 2016 , como se observa en los Gráficos 6 y 7.
A continuación, se presenta la caracterización del mercado minorista de carburantes de automoción desde el ángulo de las estaciones automáticas de la CAM con base en el Estudio y se resaltan sus resultados principales en cuanto al efecto de la entrada de las automáticas sobre la fijación de precios de las estaciones atendidas en su entorno.

Los mecanismos de fijación de precios presentan un fuerte carácter local en el mercado minorista de carburantes, donde los productos son homogéneos y difícilmente sustituibles -al menos a corto plazo-, y los consumidores repostan sus vehículos en el trayecto entre sus hogares y su lugar de trabajo. No obstante, por el lado de la oferta existe sustituibilidad a nivel local, ya que la gran mayoría de EE SS dispone de los mismos tipos de carburantes. En consecuencia, uno de los factores principales de diferenciación entre EE SS es su ubicación.

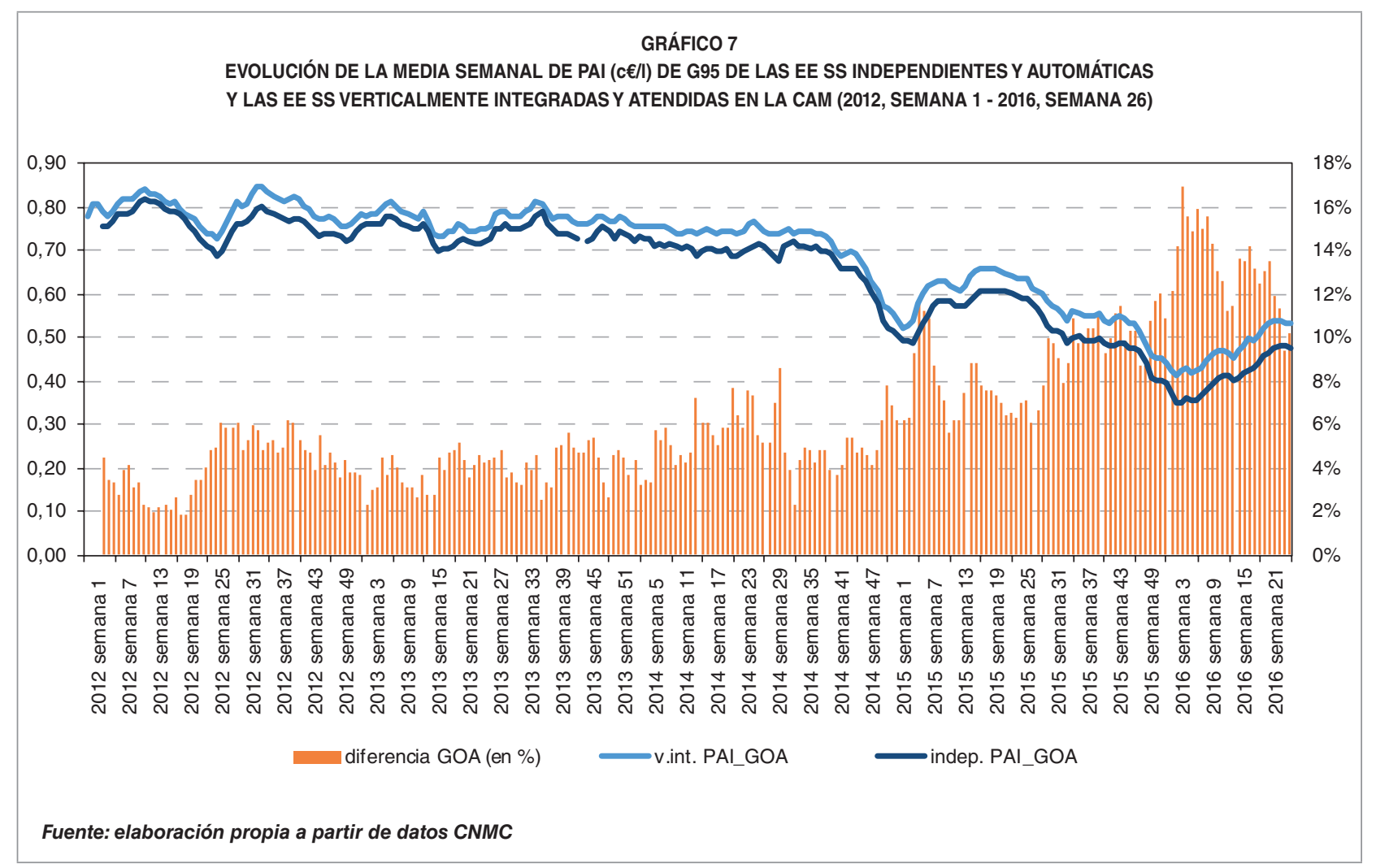


La determinación de esta zona de influencia local $^{7}$ (catchment area), en este caso establecida mediante isócronas de 10 minutos, es crucial en la estructura del Estudio, ya que determina qué estaciones se considerarán afectadas por la entrada de una estación automática y cuáles no.

La cuantificación del impacto de las estaciones automáticas sobre los precios de las estaciones atendidas la realiza el Estudio en un marco de estimación con diferencias en diferencias (en adelante DID). Esta metodología permite comparar la evolución de los precios de aquellas EE SS que han sufrido el shock (grupo de tratamiento) con la de las estaciones que no han experimentado la entrada de una automática en una isócrona de 10 minutos. El Estudio estima tres variantes del modelo DID: el modelo base, el modelo con interacción y el modelo switch.

El resultado principal del modelo base ${ }^{8}$ del Estudio es que las automáticas inducen una mayor competencia al reducir los precios de sus rivales en los mercados locales. En concreto, tras considerar las variables de control $^{9}$, la entrada de una EE SS automática

\footnotetext{
En la literatura tanto nacional (e.g., Pedriguero y Borrell, 2018; Bernardo, 2017) como internacional (e.g. Barron et al., 2004; Kim y Kim, 2010; OFT, 2014; Decisión de la Comisión Europea M.7849, 2016b; y la CNMC, 2017a y b) existe una gran variedad de definiciones en cuanto a las distancias que pueden delimitar el mercado relevante.

$8 \ln \left(\right.$ pai $\left._{i t}\right)=\alpha_{0}+\sum_{i=1}^{618} \mu_{i} \cdot E E S S_{i}+\sum_{t=1}^{260} \lambda_{t}+\beta \cdot D i D_{i t}+\theta \cdot X_{i t}+\varepsilon_{i t}$, donde la variable explicada, $\ln \left(\right.$ pai $\left._{i t}\right)$, es el logaritmo neperiano de los PAI de los carburantes en las EE SS del grupo de tratamiento en la semana $t$. Se realizan dos estimaciones distintas: una sobre los precios de GOA y otra sobre los precios de G95. Mediante los vectores de EE SS con 618 dummies para cada EE SS, y de semana $a_{t}$ con 260 dummies, sus coeficientes $\mu_{i}$ y $\lambda_{t}$ captan los efectos fijos de las EE SS que permiten controlar los efectos no observables y constantes en el tiempo.

La variable de interés es $D i D_{i t}$ que es una variable binaria clave que toma valor 1 si en la semana $t$ la estación de servicio $i$ tiene una estación automática en una isócrona de 10 minutos, y 0 en caso contrario. Así, el coeficiente, $\beta \cdot 100$, informa del efecto porcentual medio en el precio del carburante provocado por la entrada de una estación de servicio automática en una zona de influencia local.

$X$ es una matriz con variables de control que pueden influir en la fijación de precios de las EE SS y que están relacionadas con el entorno de competencia local.

9 Estas variables de control recogen las siguientes características: a) el tipo de operador (verticalmente integrado, otro abanderado o
}

hace que el PAI de GOA y G95 de las estaciones cercanas (a una distancia de 10 minutos en coche) caiga de media un $0,48 \%$ y un $0,21 \%$, respectivamente. El segundo resultado destacable es que el tipo de operador influye en el nivel de precios, es decir, las EE SS independientes tienen de media precios inferiores en ambos carburantes (GOA: $-3,64 \%$; G95: $-2,56 \%$ ) con respecto a las verticalmente integradas.

En tercer lugar, se verifica que el número de rivales en el mercado local es importante para la competencia en precios. Cada EE SS independiente adicional contribuye a la reducción de precios, de media, en un 0,38\% (GOA) y un $0,29 \%$ (G95). El hecho de que los rivales de operadores verticalmente integradas y otras abanderadas no influyan sobre los precios (coeficientes estadísticamente no significativos) refuerza la importancia de que las EE SS sean independientes para así alcanzar mayores niveles de competencia.

Por último, el modelo base arroja otros resultados interesantes. En localidades pequeñas o medianas los precios son menores que en las grandes ciudades. Un mayor consumo eléctrico municipal, variable que pretende aproximar la actividad económica de la zona, podría apuntar a que en zonas más industrializadas existe mayor competencia en precios. Además, en línea con la literatura ${ }^{10}$, la localización adyacente a una autopista o autovía produce un aumento de precios de un 1\% (GOA) y un $0,9 \%$ (G95).

\footnotetext{
independiente); b) el máximo número de rivales independientes y no independientes de cada estación de servicio en una isócrona de 10 minutos en cada año; c) la ubicación de las EE SS (mediante las siguientes variables binarias: si la estación se encuentra al lado de una carretera o autopista, y si está ubicada en una ciudad grande, mediana o una localidad pequeña); d) los servicios complementarios ofrecidos por las EE SS (mediante variables binarias), como pueden ser lavado, agua \& aire, tienda y cafetería; y e) el consumo eléctrico anual (TWh) del municipio en el que se ubica la estación, para aproximar la actividad económica de este.

10 Bruzikas et al., 2014.
} 
Los modelos DID_interacción ${ }^{11} 12$ del Estudio demuestran que las EE SS atendidas independientes son las más sensibles a la entrada de automáticas. Así, estos modelos completan al anterior (modelo base) incluyendo cada uno la variable binaria que recoge el tipo de compañía afectada por el shock (tcomp) y la interacción de esta con la variable DID (tcomp·DID) ${ }^{13}$. De esta forma, se realizan tres estimaciones paralelas, distinguiendo los tres tipos de operadores considerados: verticalmente integrados, otras abanderadas e independientes.

Los resultados de la estimación del modelo DID_interacción corroboran que las EE SS atendidas independientes tienen más flexibilidad y libertad para ajustar sus precios a los condicionantes del mercado. En concreto, la entrada de una automática provoca el mayor efecto sobre los precios de las independientes, las cuales reducen de media sus precios un $2,16 \%$ en GOA y un $1,80 \%$ en G95. Por su parte, las EE SS atendidas verticalmente integradas son las que menos reaccionan a la presencia de estaciones automáticas.

Por último, el modelo DID_switch del Estudio permite diferenciar entre los efectos competitivos inducidos por la entrada de automáticas

11 Con el fin de verificar la validez de los modelos, en el Estudio se demuestran tendencias paralelas entre la evolución de los precios de ambos grupos durante el periodo previo a la entrada de estaciones automáticas (más detalles en Bertrand et al., 2004; y Ashenfelter et al., 2013).

12 Para mitigar potenciales problemas de endogeneidad procedentes de la autorización municipal necesaria para la apertura de una estación de servicio, en el Estudio se verificó que la selección de las instalaciones, tanto en el grupo de control como en el de tratamiento, ha sido adecuada. Mediante el procedimiento de Propensity Score Matching (PSM) se escogieron aquellas estaciones en el grupo de control que, de acuerdo con determinadas variables, sean las más parecidas a las estaciones que han sufrido el shock. Tras esta verificación, se confirmó que los resultados de las estimaciones DID_interacción con y sin selección PSM son muy similares aun cuando el número de observaciones se haya reducido en un $70 \%$, como es el caso de la estimación con PSM. De esta forma queda validada la especificación del modelo DID_interacción.

13 Adicionalmente, en el Estudio se incorporan las dos variables con sus coeficientes en el modelo base:

$\ln \left(\right.$ pai $\left._{i t}\right)=\alpha_{0}+\sum_{i=1}^{618} \mu_{i} E_{E S S_{i}}+\sum_{t=1}^{260} \lambda_{t}$ semana $_{t}+\beta$ DiD $_{i t}+\gamma \cdot$ tcomp $_{i t}+\delta \cdot \operatorname{DID}_{i t}$ tcomp $_{i t}+\theta X_{i t}+\varepsilon_{i t}$ nuevas y de reconversión ${ }^{14}$. Como cabría esperar, se confirma que las estaciones automáticas nuevas conllevan mayores reducciones de precios en sus mercados locales $(0,82 \%$ en GOA y $0,52 \%$ en G95) en comparación con la entrada de estaciones reconvertidas. En cuanto a la reacción de los diferentes tipos de operadores de las estaciones atendidas y afectadas, se confirma que la presencia de las automáticas nuevas implica mayores reducciones en los precios de las independientes, que alcanzan una reducción media de un 3,5\% en caso de GOA y un 2,9\% en caso de G95. En cambio, el efecto competitivo inducido por las EE SS automáticas reconvertidas es significativamente menor y en muchos casos es inexistente.

\section{Conclusiones}

Según ha puesto de manifiesto la autoridad de competencia en varias ocasiones (CNMC, 2016 y 2019), la entrada de EE SS automáticas aumenta la presión competitiva en el mercado de distribución minorista de carburante y, por tanto, presiona a la baja los precios de los carburantes de automoción, sector estratégico para el país.

En efecto, el estudio de la CNMC (2019) realizado para las EE SS automáticas en la Comunidad de Madrid demuestra que la entrada de este tipo de instalaciones favorece la reducción de precios de los carburantes en las estaciones atendidas. Concretamente, en el periodo de los cinco años estudiados (entre $\triangleright$

14 El Estudio señala que a mediados de 2016 había un total de 69 EE SS automáticas en la CAM, de las cuales 41 eran nuevas, mayoritariamente operadas por empresas independientes, y 28 reconvertidas de estaciones atendidas en automáticas, que principalmente han sido operadas por empresas verticalmente integradas, como Repsol, Cepsa o BP. 
mediados de 2011 y 2016), aquellas EE SS atendidas que tenían cerca una estación de servicio automática bajaron sus precios de media un $0,5 \%$ para el gasóleo A y un 0,2\% para la gasolina 95 en los seis meses siguientes a la entrada de la estación de servicio automática. Descomponiendo este efecto entre las diferentes estaciones atendidas en función del tipo de operadores, según sean independientes, verticalmente integrados $u$ otros abanderados, se concluye que son las estaciones atendidas independientes las que más reaccionan a la entrada de sus rivales automáticas.

Es de esperar que, de forma parecida a lo experimentado en la CAM, las estaciones de servicio automáticas induzcan mayor competencia en otras comunidades autónomas, especialmente donde su penetración actualmente es más reducida.

Por tanto, dado que la presencia de EE SS automáticas genera presiones procompetitivas que permiten disciplinar el mercado vía precios, es importante que la regulación de dichas estaciones no sea restrictiva, sino que vaya encaminada a garantizar la seguridad y la protección de los derechos de los consumidores y usuarios, sin incorporar medidas desproporcionadas e innecesarias que conlleven la prohibición de facto de este tipo de instalaciones (al exigir de manera directa o indirecta la presencia física de personal). Una regulación apropiada en este sentido no tiene por qué suponer la desaparición de las EE SS tradicionales, sino que permitirá la convivencia de ambos modelos de negocio, pudiéndose además prestar un servicio más cercano a zonas rurales o alejadas de los núcleos urbanos. Todo ello redundará en beneficios para el consumidor, que dispondrá de un mayor abanico de posibilidades en un sector clave para la economía.

\section{Bibliografía}

Ashenfelter, O.C., Hosken, D.S., y Weinberg, M.C. (2013). The Price Effects of a Large Merger of Manufacturers: A Case Study of Maytag - Whirlpool. American Economic Journal: Economic Policy 2013, 5(1): 239-261. http://dx.doi.org/10.1257/pol.5.1.239

Asociación Nacional de Estaciones de Servicio Automáticas (AESAE) (2019). Estudio de mercado EE SS automáticas, Carter Lane. Estudio presentado en Madrid el 2 de julio de 2019 en el primer encuentro de estaciones de servicio automáticas.

Barron, J.M., Taylor, B.A., y Umbeck, J.R. (2004). Number of sellers, average prices, and price dispersion. International Journal of Industrial Organization, 22(8-9), 1041-1066.

Bernardo, V. (2017). The effect of entry restrictions on price. Evidence from the retail gasoline market. https://editorialexpress.com/cgi-bin/conference/ download.cgi?db_name=RESJunSymp2016\& paper_id=217

Bertrand, M., Duflo, E., y Mullainathan, S. (2004). How much should we trust difference-in-differences estimates? The Quarterly Journal of Economics, 119(1), 249-275.

Bruzikas, T., y Soetevent, A. (2014). Detailed data and Changes in Market Structure: The move to Unmanned Gasoline Service Stations, University of Groningen, 14027-EEF.

Comisión Europea (2003). Case M.3291 - Preem Skandinaviska Raffinaderi.

Comisión Europea (2008). Case No COMP/M.5005 - Galp Energia / Exxonmobil Iberia.

Comisión Europea (2015). Expost analysis of two mobile telecom mergers: T-Mobile/tele.ring in Austria and T-Mobile/Orange in the Netherlands.

Comisión Europea (2016a). Case M.7603 - Statoil Fuel And Retail / Dansk Fuels.

Comisión Europea (2016b). Case M.7849 - Mol Hungarian Oil And Gas / Eni Hungaria / Eni Slovenija. 
CNMC (2016). Propuesta referente a la regulación del mercado de distribución de carburantes de automoción a través de estaciones de servicio desatendidas (PRO/CNMC/002/16).

CNMC (2017a). Informe y Propuesta de Resolución, Expediente C/0835/17 CEPSA/Villanueva/Paz.

CNMC (2017b). Informe y Propuesta de Resolución, Expediente C/0890/17 DISA/GESA.

CNMC (2019). Análisis del efecto competitivo de la entrada de gasolineras automáticas en el mercado de distribución minorista de carburantes (E/CNMC/005/2019).

Drivkraft Danmark (2019). Energistatistik 2019. https://www.drivkraftdanmark.dk/wp-content/ uploads/2019/05/DD_Energistatistik_2019_ WEB-spreads.pdf
Ministerio de Industria, Comercio y Turismo (2019). Guía Técnica de Aplicación Práctica de la Instrucción Técnica Complementaria MI-IP de Instalaciones para el Suministro de Vehículos. Dirección General de Industria y la PYME (enero de 2019). http://www.f2i2.net/documentos/lsi/InstPetroliferas/Guia_Aplicacion_IP_04_2019.pdf

Pedriguero, J., y Borrell, J.R. (2018, may). Driving Competition in Local markets with Near-Perfect Substitues: an Application on the Spanish Retail Gasoline Market. Empirical Economics. http:// doi.org/10.1007/s00181-018-1427-6

Svenska Petroleum \& Biodrivmedel Institutet (SPBI) (2019). https://spbi.se/statistik/forsaljningsstallen/

US Department of Justice y la Federal Trade Commission (2010). Horizontal Merger Guidelines. 
